\title{
Application of «Sensor signal analysis network» complex for distributed, time synchronized analysis of electromagnetic radiation
}

\author{
Vladimir Mochalov ${ }^{1, *}$, Anastasia Mochalova ${ }^{1}$ \\ ${ }^{1}$ Institute of Cosmophysical Research and Radio Wave Propagation FEB RAS, 684034 Kamchatka \\ region, Elizovskiy district, Paratunka, Russia
}

\begin{abstract}
The paper considers a developing software-hardware complex «Sensor signal analysis network» for distributed and time synchronized analysis of electromagnetic radiations. The areas of application and the main features of the complex are described. An example of application of the complex to monitor natural electromagnetic radiation sources is considered based on the data recorded in VLF range. A generalized functional scheme of stream analysis of signals by a complex functional node is suggested and its application for stream detection of atmospherics, whistlers and tweaks is considered.
\end{abstract}

\section{Introduction}

At the present time authors are developing a new software-hardware complex «Sensor signal analysis network» (SSAN) for construction and functioning of sensor networks for distributed analysis of time synchronized signals. In this paper we accept that a sensor network is a distributed network of endpoint functional nodes (F-nodes) which analyze the time synchronized signals and transmit the collected information (analysis results) to one or several collecting centers. In general form, information can be transmitted both via the wire and wireless networks. In case of application of wireless networks, owing to the selforganization property and application of special protocols, it becomes possible to transmit the information by retransmission from one node to another using the transit nodes (T-nodes) (Fig.1)

Fig. 2 shows a generalized scheme of SSAN complex F-node, including a mini-PC, measurement time synchronization unit, ADC, sensors, communication modules and optional field-programmable gate array (FPGA). In case of the absence of the necessity to use the FPGA, the ADC module is connected directly to a mini-PC. Depending on the conditions of application and accuracy requirements, the time synchronization unit may be realized on the basis of application of different types of satellite time synchronization GPS/GLONASS modules (NMEA 0183 signal comes to a mini-PC, PPS signal comes to ADC and FPGA input, the stabilized output of $10 \mathrm{MHz}$ from GPS/GLONASS module comes to FPGA input) and/or the connectives of temperature-stabilized precise highlyreliable quartz oscillator with FPGA.

Corresponding author: vmochalov@ikir.ru 


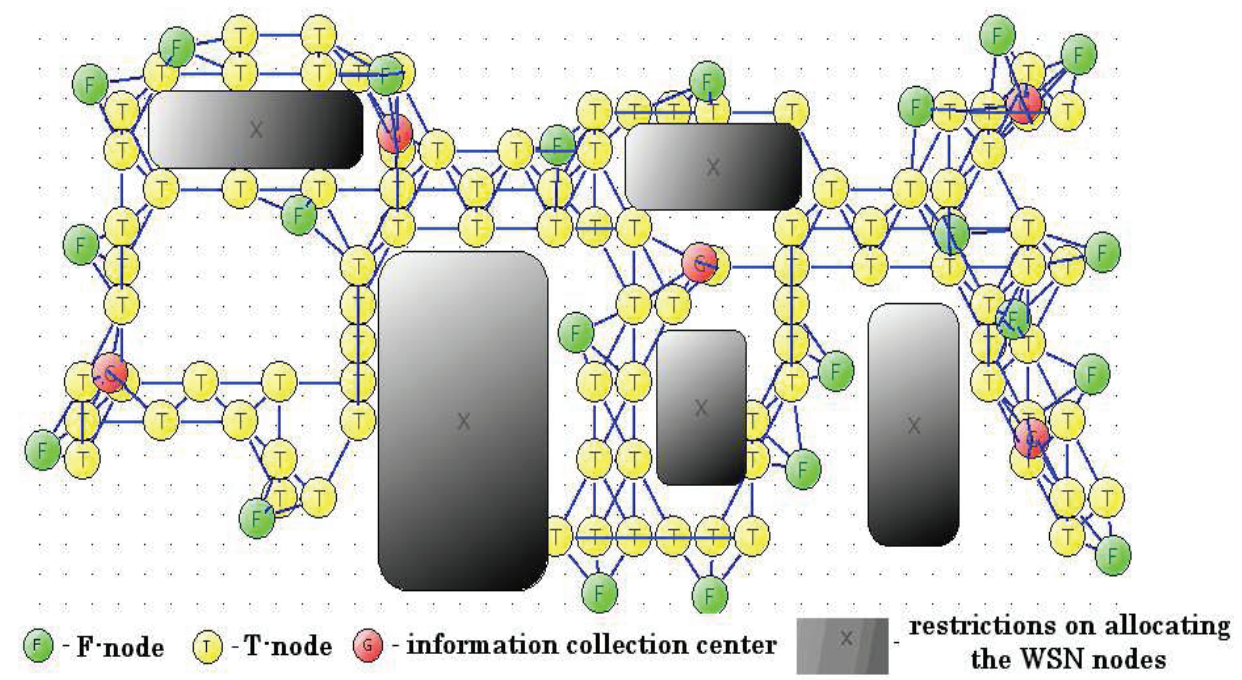

Fig. 1. Example of a distributed fail-tolerant system structure of a wireless sensor network [1].

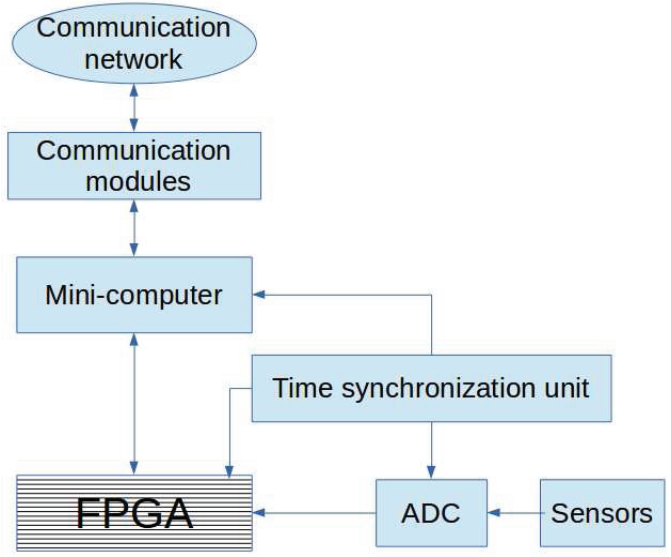

Fig. 2. Functional scheme of F-node of the software-hardware complex «Sensor signal analysis network».

Scope and field of application of SSAN complex:

- Projecting of sensor network structure taking into account different variants of network node location (ground, ocean, drones and spacecrafts),

- Application of universal software-hardware mini-PC nodes with time synchronization for fast implementation of sensor networks for the purpose of distributed analysis of time synchronized signals,

- Distributed complex analysis of electromagnetic radiation to investigate the magnetosphere dynamics, synoptic weather systems, seismic, volcanic activity etc.

Potential users of SSAN complex:

- The software-hardware complex can be applied for scientific investigations as well as for monitoring and automation of distributed object control, life-supporting system automation, detection and prevention of emergency situations, road monitoring (including railway roads, oil and gas pipe-lines, electrical and heat supply networks etc.) and so on.

SSAN complex features:

- Unique software has been developed [2] which allows us to build a distributed faulttolerant sensor network structure in cooperation with a designer and to optimize the 
network node arrangement. Based on the developed algorithms [1, 3], the software arranges monitoring network nodes on a plane taking into account spatial limitations for node location and suitability evaluation functions. In the result of software operation, a list with the coordinates of distributed monitoring network nodes is formed and the image of network node arrangement is presented,

- The possibility to connect user modules for signal analysis, low cost of hardware for the mini-PCs of network computational nodes and low power consumption ( $\sim 5 \mathrm{Watt}$ ) allow us to apply the hardware-software complex to solve a wide range of problems of distributed analysis of time synchronized signals,

- Application of a ready-made configured software-hardware complex will reduce organization facilities for the solution of some problems in registration and analysis of time synchronized signals.

\section{Functional scheme of signal stream analysis by SSAN complex F-node on the example of monitoring of natural electromagnetic radiation sources based on the data of VLF range registration}

The paper [4] describes one of the ways of realization of SSAN F-node (Fig. 3) which is used for monitoring of natural electromagnetic radiation sources based on the data of VLF range registration.

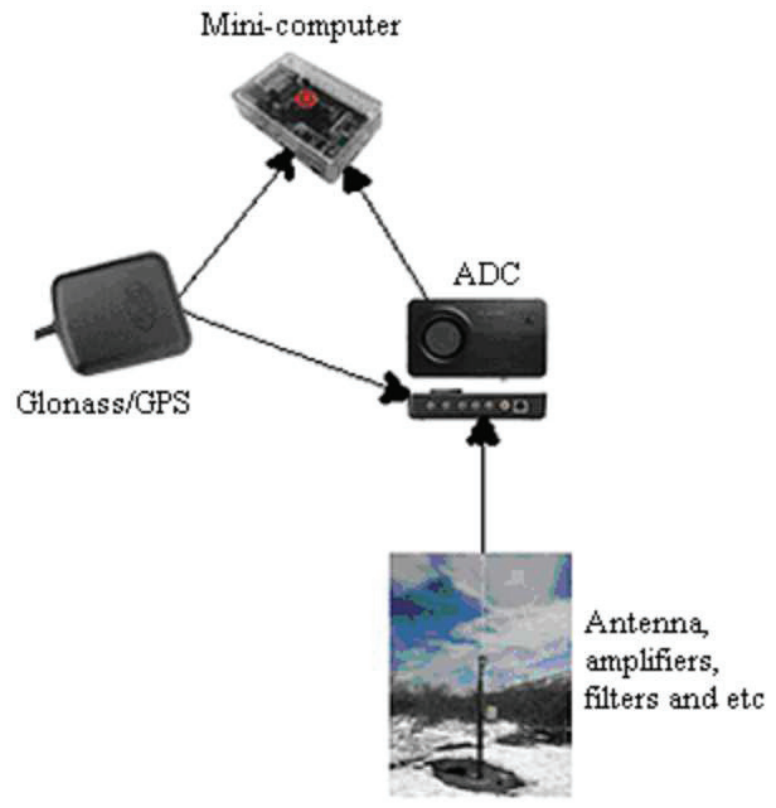

Fig. 3. Example of F-node for monitoring of natural electromagnetic radiation sources based on the data of VLF range registration [4].

Signal from the antenna comes to a pre-amplifier and then to a sound card linear input (applied as one of ADC variants). PPS signal from Glonass/GPS module also comes to the sound card linear input that allows us to make time synchronization of the measurements at different sensor network F-nodes. NMEA 0183 signal comes to a mini-PC to set the system time and to determine the F-node location. After digitization, the general signal from the sound card linear input comes to the mini-PC for stream processing. Several programs are 
launched on the mini-PC working under Linux operating system, they are: 1) program for data reading from $\mathrm{ADC}$; 2) program of stream analysis (SA) of natural electromagnetic radiation sources based on the data registered in VLF range. SA program is capable of operating in two modes: 1) read mode of digitized data on different channels into a circular buffer inner structure and custom actions during every read record (almost real-time processing mode); 2) record mode of files with a given duration with the following processing of files by custom actions.

Fig. 4 shows a generalized functional scheme of signal stream analysis by SSAN complex F-node. Depending on the applied algorithms for event recognition, the following actions can be performed at the stage of primary processing: data normalization, calculation of signal spectrogram with different FFT window sizes (Fig. 5 and 7) and in different scales (linear and logarithmic) (Fig. 6 and 8), data filtration from noise (Fig. 9), estimation of different wavelet transforms (Fig. 10) and so on.

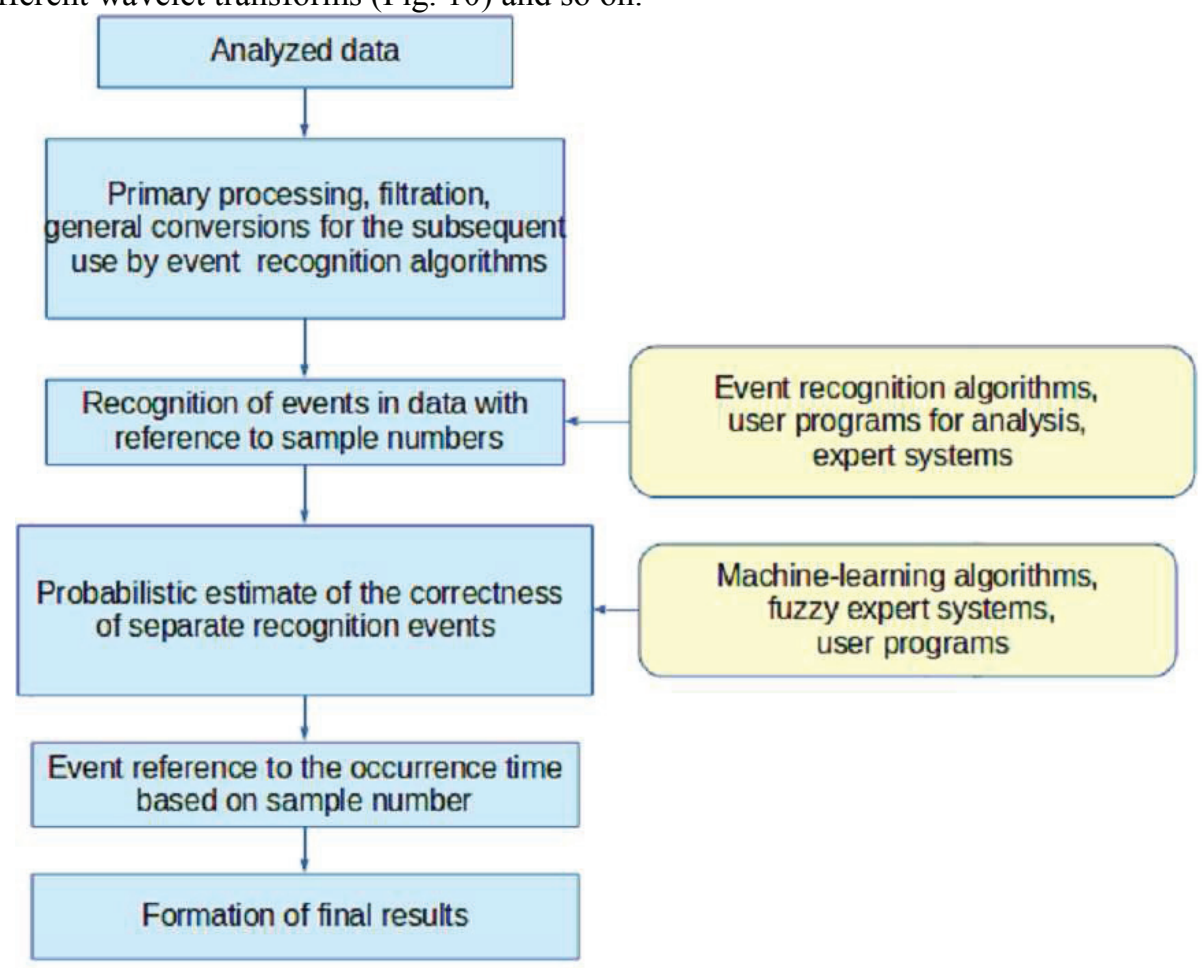

Fig. 4. Generalized functional scheme of signal stream analysis by SSAN complex F-node.

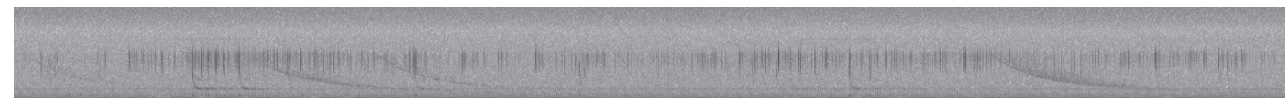

Fig. 5. Example of a spectrogram in linear scale on frequency with FFT window size of 256 and sampling rate of $44100 \mathrm{~Hz}$. Whistlers, tweaks and atmospherics are seen on the spectrogram.

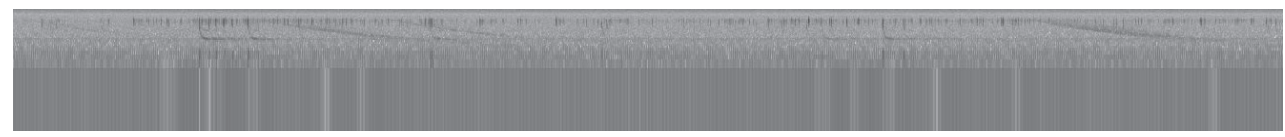

Fig. 6. Example of a spectrogram in logarithmic scale on frequency with FFT window size of 256 and sampling rate of $44100 \mathrm{Gz}$. Whistlers, tweaks and atmospherics are seen on the spectrogram. 


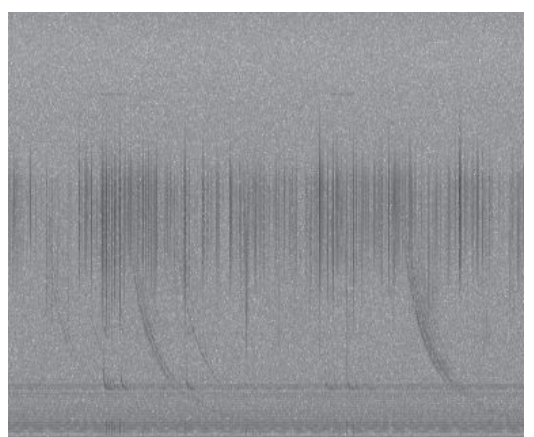

Fig.7. Example of a spectrogram in linear scale on frequency with FFT window size of 1024 and sampling rate of $44100 \mathrm{~Hz}$.

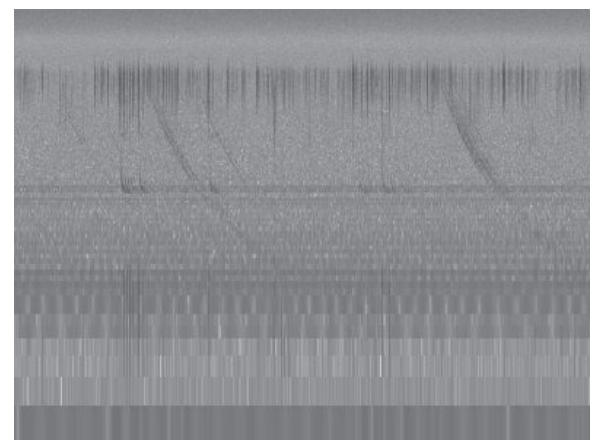

Fig.8. Example of a spectrogram in logarithmic scale on frequency with FFT window size of 1024 and sampling rate of $44100 \mathrm{~Hz}$.

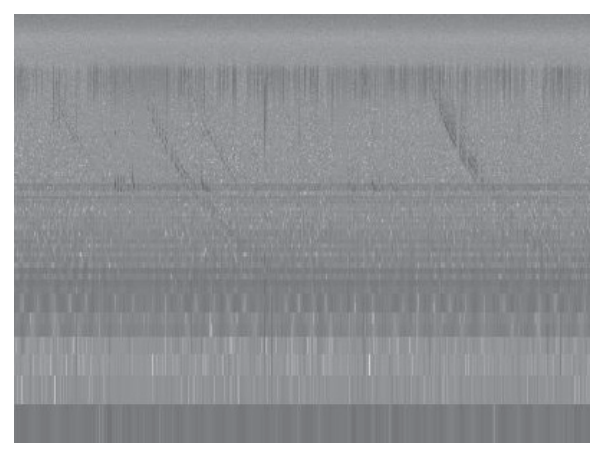

Fig.9. Example of a spectrogram filtered from strong atmospherics.

After the preliminary processing, filtration and other conversions, algorithms for event recognition (for example, atmospherics, tweaks and whistlers) are implemented. Different algorithms are applied to recognize different types of events. For example, a whistler can be recognized by a template (in the simplest case it is a line segment with different inclination angles) on a spectrogram filtered from strong atmospherics and plotted in a logarithmic scale on frequency. To recognize an atmospheric, we can apply analysis of its wave form (shape, amplitude, number of zero level intersections etc.), spectrogram analysis at different scales of FFT windows, hybrid approach of joint analysis of a wave form and a spectrogram and so on. 


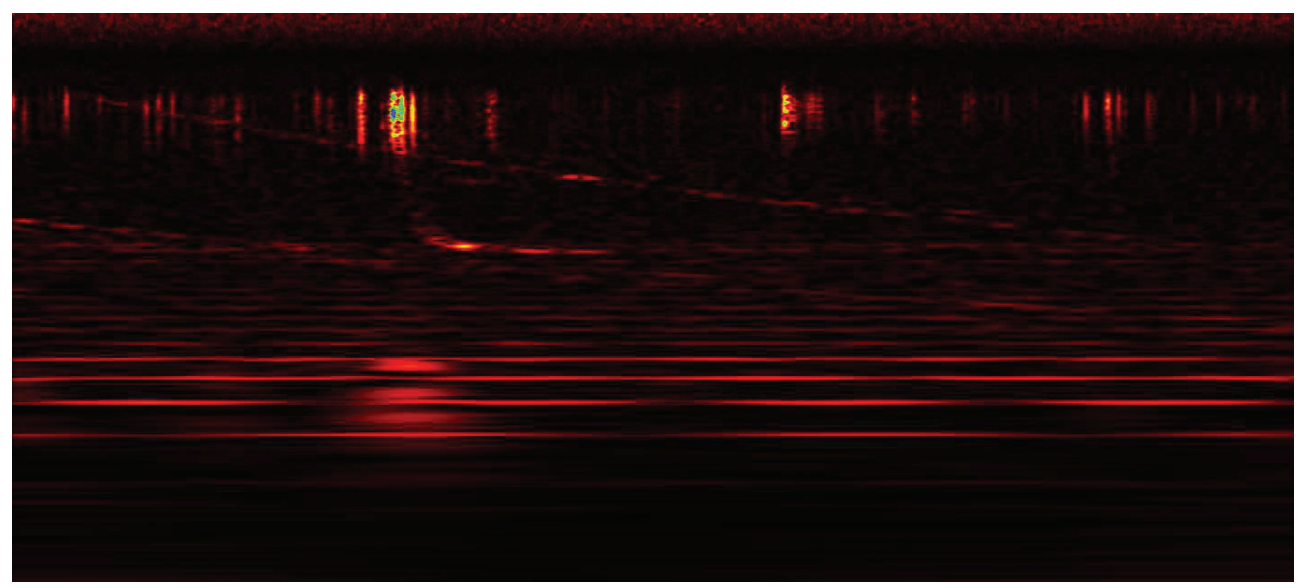

Fig.10. Example of continuous wavelet-transform with Morlet wavelet. Atmospherics, a long whistler and a tweak are clear in the figure.

Each algorithm of event recognition can be characterized by different criteria: operation quality (the probability of correct or false detection of an event, estimate of the number of correctly recognized events from the total number, stability against noises etc.), applied classification model and the necessity to train it, flexibility and restrictions of application, operation rate, main memory footprint and so on. It is reasonable to built the system for stream analysis of different signals in analogy with hybrid intrusion detection systems where expert system are used together with different algorithms of intrusion and attack detection. For example, having different expert rules and prepared program modules for event recognition, it becomes possible to generalize the results of operation of different algorithms, to make specialized data filtration (for example, removal of atmospherics and tweaks for better whistler recognition), to make probabilistic estimate of separate event detection correctness (applying, for example, fuzzy expert systems, machine learning algorithms) and so on. After generalization of the results of different algorithm operation, the detected events are bound to sample numbers, final results of the analysis are formed and the data are sent to the collecting centers.

\section{Acknowledgments}

The work was supported by the VarSITI Grant "Creation of a database for atmospheric and whistler events detected in the Russian Far East".

\section{References}

1. V.A. Mochalov, Lecture Notes in Computer Science, 9247, 11-22 (2015).

2. V.A. Mochalov, Certificate of registration of computer software, 2016612039 (2016).

3. V. Mochalov, Proceedings, The IEEE 17th International Conference on Advanced Communication Technology, 34-42 (2015).

4. V. Mochalov, D. Sannikov, R.Karimov, B.Shevtsov, G. Drugin, N. Cherneva, A. Mochalova, J. Lichtenberger, V. Argunov, Procedia Engineering, 168, 1721-1724 (2016). 